\title{
A!
}

This is an electronic reprint of the original article.

This reprint may differ from the original in pagination and typographic detail.

Ouahouah, Sihem; Prados-Garzon, Jonathan; Taleb, Tarik; Benzaid, Chafika

\section{Energy-aware Collision Avoidance stochastic Optimizer for a UAVs set}

Published in:

2020 International Wireless Communications and Mobile Computing, IWCMC 2020

DOI:

10.1109/IWCMC48107.2020.9148495

Published: 01/06/2020

Document Version

Peer reviewed version

Please cite the original version:

Ouahouah, S., Prados-Garzon, J., Taleb, T., \& Benzaid, C. (2020). Energy-aware Collision Avoidance stochastic Optimizer for a UAVs set. In 2020 International Wireless Communications and Mobile Computing, IWCMC 2020 (pp. 1636-1641). [9148495] (International Wireless Communications and Mobile Computing Conference). IEEE. https://doi.org/10.1109/IWCMC48107.2020.9148495

This material is protected by copyright and other intellectual property rights, and duplication or sale of all or part of any of the repository collections is not permitted, except that material may be duplicated by you for your research use or educational purposes in electronic or print form. You must obtain permission for any other use. Electronic or print copies may not be offered, whether for sale or otherwise to anyone who is not an authorised user. 


\title{
Energy-aware Collision Avoidance stochastic Optimizer for a UAVs set
}

\author{
Sihem Ouahouah *\|, Jonathan Prados-Garzon $\|$, Tarik Taleb $\| \S$ and Chafika Benzaid $\|$ \\ \| Aalto University, Espoo, Finland. Emails: first-name.last-name@aalto.fi \\ $\S$ University of Oulu, Oulu, Finland \\ * Ecole nationale Supérieure d'Informatique, Algiers, Algeria. Email: s_ouahouah@esi.dz
}

\begin{abstract}
Unmanned aerial vehicles (UAVs) is one of the promising technology in the future. A recent study claims that by 2026 , the commercial UAVs, for both corporate and customer applications, will have an annual impact of 31 billion to 46 billion on the country's GDP. Shortly, many UAVs will be flying everywhere. For this reason, there is a need to suggest efficient mechanisms for preventing the collisions among the UAVs. Traditionally, the collisions are prevented using dedicated sensors, however, those would generate uncertainty in their reading due to their external conditions sensitivity. From another side, the use of those sensors could create an extra overhead on the UAVs in terms of cost and energy consumption. To deal with these challenges, in this paper, we have suggested a solution that leverages the chance-constrained optimization technique for avoiding the collision in an energy-efficient manner. Building on the expressions for the non-central Chi-square CDF and expected value, and through the convexification of the resulting expressions, the chance-constrained optimization program is transformed into a convex Mixed Binary Nonlinear one. The resulting program allows us to find the optimal safety distance that extends UAVs life-time and allows every UAV to move with a guaranteed probability of collision between any pair of UAVs.
\end{abstract}

\section{INTRODUCTION}

Unmanned aerial vehicles (UAVs) have been recently gaining momentum due to their high flexibility, low cost as well as their multidisciplinary use[1]. The Flight American Federation (FAF) envisions that about 2 million UAVs will be in use by 2022. However, the proliferation of UAVs will come with unavoidable challenges, such as collision menaces. In this work, we are focusing on how to avoid collisions among UAVs during their journeys.

Over the previous years, several solutions have been proposed to tackle the UAVs collision problem. Most of those solutions [2]-[4] have adopted a two stages approach namely, sensing and avoiding [5]. The first stage consists in localizing the coordinates of any intruder getting closer to a UAV that carries a sensor equipment (e.g., camera,radar, etc.). Meanwhile, the second stage consists in maneuvering a UAV to a safe localization which is geometrically computed based on intruder's coordinates. Despite its simplicity, sense and avoid collision approach exhibits the following limitations: $i$ ) the high sensitivity of sensors to external-conditions (e.g., weather perturbations, noises, etc.) could impact negatively on their accuracy, then UAV collision avoiding process could be negatively impacted too; $i i$ ) the use of resource-greedy sensors like camera could impact negatively on UAV's battery life-time; $i i i)$ the high cost of sensors with high accuracy (e.g.LIDAR); $i v$ ) the collision avoidance system, usually,is limited only to two UAVs.
To address the problem of collision among a UAVs set, authors in [6] have suggested EDC-UAV solution. EDC-UAV allows to avoid collisions while UAVs life-time is extended. Although EDC-UAV considers GPS's margin error, it assumes that the error is uniformly bounded within a circle referred by its radius. However, it has been proven that GPS's margin error follows a Gaussian distribution [7], which makes the assumption of EDC-UAV unrealistic and UAVs could have an extra traveled distance which probably impact negatively on their energy capacity. To avoid such issue, new solutions [8], [9], have recently leveraged a stochastic approach aiming to devise a practical UAV collision avoidance system. For instance, authors in [8] have proposed a chanceconstraint based (i.e., stochastic-based) optimization for self path-planning while avoiding collisions with static obstacles. Unfortunately, the suggested solution considers only a single UAV. In [9], a chance-constrained solution is proposed to avoid collision in a multi-agent system while taking into account both static and dynamic obstacles. Then, they have designed a model that supports only two agents that has been tested with a scenario of two UAVs. This solution ensures that the euclidean distance probability between a pair of UAVs is less than a given probability. Since the derivation of a chance-constrained is quietly hard, authors in [9] have been transformed their problem to deterministic one using,only, the expected value which is a precision-less method.

In this paper, we aim to overcome aforementioned limitations by suggesting an energy-aware collision avoidance solution among a UAVs set, and by considering realistic collision scenario (i.e, GPS's error follows a Gaussian distribution). To this end, we devise a chance-constrained optimization model that could be deployed either by a groundcontroller or a mobile-controller (e.g., one of the UAVs or an edge-controller). Unlike the previous cited solutions, which use a predefined value of the threshold distance used to increase the UAV safety. Our solution gives a new definition to the threshold distance by considering that a collision between any pair of UAVs is likely to happen when the distance between them is closer to their dimensions.

Furthermore, in contrast to [9], based on the expressions for the non-central Chi-square CDF [10] and expected value, and through the convexification of the resulting expressions, the chance-constrained optimization program is transformed into a convex Mixed Binary Nonlinear one. The resulting program allow us to find the optimal safety distance that extends UAVs life-time and allows every UAV to move with 
a guaranteed probability of collision.

The rest of the paper is organized as follows. In section II a related work is presented. In section III our system model and problem formulation are provided. In section IV our solution description, derivation and performances are presented. Finally, conclusions are drawn in section VI.

\section{RELATED WORK}

The problem of collision avoidance has been addressed in the context of ground vehicles [11], [12] as well as in the context of UAVs [2]-[4], [13]-[21]. Some solutions leverage on camera sensors to detect obstacles around the UAV [3], [4]. Nevertheless, the information provided by camera sensors entails intensive processing in order to be converted into useful data to control UAVs [5], making cameras inappropriate for real-time applications. Similarly, the use of radar, as in [2], is unsuitable for small-scale UAVs due to its size and weight [5]. Moreover, most of the aforementioned solutions assume that data captured by sensors and GPS is error-free. However, in real-world scenarios such assumption cannot be fulfilled due to external conditions (e.g., weather conditions) affecting the precision of sensors and GPS. Authors in [6] proposed EDC-UAV; a solution to avoid collisions within a swarm of UAVs while taking into account the margin error in GPS localization. Although EDC-UAV considers GPS's margin error, it assumes that the error is bounded following a uniform distribution. The error uniformity assumption is also unrealistic as the GPS error tends to be Gaussian distributed [7]. Thus, a probabilistic characterization of the uncertainty in GPS localization is necessary to provide practical collision avoidance solutions. Chance-constrained approach has been leveraged to formulate problems under stochastic uncertainty (e.g., ). Blackmore et al. [8] used a chance-constrained approach to plan autonomous aircraft trajectory while avoiding obstacles. The authors focused on one UAV and static obstacles. The work in Kothari13 proposes a real-time chance constrained solution for path planning of a single UAV. Unlike [8], both static and dynamic obstacles are considered.Lyons et al. [9] formulated the problem of a chance-constrained model predictive control for multi-agent systems then the transformation model is designed only for two agents. Furthermore, the authors [9] have transformed their model based on the expected-value derivation which is precision-less method.

\section{SYSTEM MODEL AND PROBLEM FORMULATION}

\section{A. System model}

Let us assume that we have a set of $K$ UAVs fulfilling the same mission $M$ and move under the control of the the same operator. During $M$, every UAV $U_{k} \in K$ moves following a straight-line path within a confined area of dimension $X \times Y$. Independently of the UAVs' shapes, we assume that their shapes are inscribed in their circle of radius $r$. Every UAV $U_{k}$, starts $M$ from an initial position $\mathcal{S}_{k}=\left(x_{k}^{0}, y_{k}^{0}\right)$ to reach a target destination $\mathcal{E}_{k}=\left(x_{k}^{T}, y_{k}^{T}\right)$. The time-duration $T$ of $M$ is incremented progressively and equitably at every time-step $t$ by adding a time-slot $\tau$ until every UAV $U_{k}$ reaches its target. We denote $V_{k}^{t}$ a UAV $U_{k}$ velocity at a time-step $t$. Every $V_{k}^{t}$ is designed as vector with $x$-axis $V_{k, x}^{t}$ component and $y$-axis $V_{k, y}^{t}$ component where, $V_{k}^{t}=V_{k, x}^{t}+V_{k, y}^{t}$ which assumed not exceeding a maximum allowable velocity Vmax. In addition, we consider that a controller tracks UAVs' motions by receiving at each timestep $t$ their GPS localization $L_{k}^{t}=\left(x_{k}^{t}, y_{k}^{t}\right)$ for the UAV $U_{k}$. Based on $L_{k}^{t}$ value and within a negligible time in $\tau$, the controller computes the position where UAVs should move safely with minimal energy consumption at the next timestep $t+1$.The UAV's safety is considered by keeping, at each time-step $t$, a safety distance threshold $D_{t h}$ between each pair of UAVs. Moreover, the UAV's energy saving is considered by reducing the traveled distance.

According to [7], the GPS sensor provides uncertain data, following a Gaussian distribution. Thus, we assume that for a GPS localization $L_{k}^{t}$, the $x$-axis coordinate, $x_{k}^{t}$ is modelled as a Gaussian random variable with standard deviation $\sigma_{k, x}$ and mean $\mu_{k, x}$; i.e., $x_{k}^{t} \sim \mathcal{N}\left(\mu_{k, x}, \sigma_{k, x}^{2}\right)$. Similarly, $y$-axis coordinate, $y_{k}^{t}$ is modelled as a Gaussian random variable with standard deviation $\sigma_{k, y}$ and mean $\mu_{k, y}$; i.e., $y_{k}^{t} \sim \mathcal{N}\left(\mu_{k, y}, \sigma_{k, y}^{2}\right)$.

\section{B. Problem formulation}

In this section, we will formally formulate our collision avoidance problem for a set of $K$ UAVs that move in a stochastic environment. In literature, when distance between two UAVs $U_{k}$ and $U_{l}$ is less than $D_{t h}$, it means that the instance of a collision threat occurs and probably the collision can not be avoided. Indeed, the probability of collision is the probability that distance between UAVs is less than $D_{t h}$. Formally, it is defined as follows:

$$
P_{r}(\text { Collision })=P_{r}\left(\left\|U_{k}, U_{l}\right\| \leq D_{t h}^{2}\right)
$$

Since the euclidean distance between two points $U_{k}$ and $U_{l}$ is defined as follows:

$$
\left\|U_{k}, U_{l}\right\|=\sqrt{\left(x_{k}-x_{l}\right)^{2}+\left(y_{k}-y_{l}\right)^{2}}
$$

where $\left(x_{k}, y_{k}\right)$ and $\left(x_{l}, y_{l}\right)$ are, respectively, the the coordinates of $U_{k}$ and $U_{l}$. Then, the equation (1) becomes as follows:

$$
P_{r}(\text { Collision })=P_{r}\left(\left(x_{k}-x_{l}\right)^{2}+\left(y_{k}-y_{l}\right)^{2} \leq D_{t h}^{2}\right)
$$

In the scenario described previously, this work addresses the collision avoidance problem that aims at allowing UAVs to accomplish their mission with a minimum energy consumption, while guaranteeing that the probability of collision between any pair of UAVs $U_{k}$ and $U_{l}$ is kept below a distance threshold $D_{t h}$. Formally, our collision avoidance problem for a set of UAVs can be formulated as follows:

$$
\begin{aligned}
\min \left\{E \left[\sum_{k=1}^{K}(\right.\right. & \left.\left(x_{k}^{t}+v_{k, x}^{t} \cdot \tau-x_{k}^{T}\right)^{2}\right) \\
& \left.\left.\left.+\left(y_{k}^{t}+v_{k, y}^{t} \cdot \tau-y_{k}^{T}\right)^{2}\right)\right]\right\}
\end{aligned}
$$


subject to:

$$
\begin{gathered}
{\left[\left(x_{k}^{t}+v_{k, x}^{t} \cdot \tau\right)-x_{k}^{T}\right]^{2}+\left[\left(y_{k}^{t}+v_{k, y}^{t} \cdot \tau\right)-y_{k}^{T}\right]^{2} \leq} \\
{\left[\left(x_{k}^{t}-x_{k}^{T}\right]^{2}+\left[\left(y_{k}^{t}-y_{k}^{T}\right]^{2} \quad \forall k \in[1, K]\right.\right.} \\
P\left\{\left[\left(x_{k}^{t}+v_{k, x}^{t} \cdot \tau\right)-\left(x_{l}^{t}+v_{l, x}^{t} \cdot \tau\right)\right]^{2}\right. \\
+\left[\left(y_{k}^{t}+v_{k, y}^{t} \cdot \tau\right)-\left(y_{l}^{t}+v_{l, y}^{t} \cdot \tau\right)\right]^{2} \\
\left.\leq\left(D_{t h}\right)^{2}\right\} \leq \epsilon \quad \forall k, l \in[1, K] \& k \neq l \\
v_{k, x}^{t}{ }^{2}+v_{k, y}^{t}{ }^{2} \leq\left(v^{k(\max )}\right)^{2}
\end{gathered}
$$

The above problem is a chance-constrained optimization program. As aforementioned, this is due to GPS uncertainty which prevents the system from having an exact knowledge of the current localization $\mathbf{L}_{k}^{t}=\left(x_{k}^{t}, y_{k}^{t}\right)$ of every UAV $U_{k}$ at each time-step $t$. As already mentioned, the coordinates $x_{k}^{t}$ and $y_{k}^{t}$ are modelled as a Gaussian random variables with parameters $\mathcal{N}\left(\mu_{x}, \sigma_{x}^{2}\right)$ and $\mathcal{N}\left(\mu_{y}, \sigma_{y}^{2}\right)$, respectively. The decision variables are the $2 \mathrm{D}$ velocity vector $\mathbf{v}_{k}^{t}=\left(v_{k, x}^{t}, v_{k, y}^{t}\right)$ for every UAV $U_{k}$. The vector $\mathbf{v}_{k}^{t}$ fully describes the motion of the UAV $U_{k}$ in the next time-step $t+1$. The problem's objective (4) is that every UAV gets as close as possible to its target in the next time-step $t+1$. In this way, the mission duration and the travelled distance of every UAV are minimized. The constraint (6) ensures that the probability of collision between any pair of UAVs $U_{k}$ and $U_{l}$ at the next time-step $t+1$ is kept below a given probability threshold $\epsilon$. Constraint (5) guarantees that every UAV moves forward to its targeted position at the next time-step. Finally, constraint (7) takes into account the speed limit of every UAV.

\section{ENERgy-AWARe Collision AVOIDANCE STOCHASTIC OPTIMIZER FOR A UAVS SET}

This section focuses on the description of the proposed Energy-Aware Collision Avoidance Stochastic Optimizer (ECASO). As stated above, ECASO aims to expand the UAVs' lifetime while ensuring that the probability of collision between UAVs is kept below a given probability threshold. Mainly, ECASO consists of two sub-modules, Kalman-Filter and optimizer. ECASO uses Kalman-Filter to localize UAVs with a high accuracy, whereas it solves the previous chance-constrained designed problem. During the mission, ECASO tracks UAVs motion by receiving at every time-step the UAV GPS localization. At that time, ECASO tests first, if all UAVs reach their targets. If it is the case, then mission accomplished and ECASO stops running. Otherwise, the Kalman-Filter applies a set of filteringfunctions to remove the noises and reduce the uncertainty from the GPS UAV's localization. Therefore, the optimizer gets the filtered localization to compute the optimal position coordinates where $\mathrm{UAV}(\mathrm{s})$ can move at the next time-step. The previous steps are repeated, at each $\tau$, until all UAVs reach their targets. More details about Kalman-Filter and the optimizer sub-modules are involved within the following sub-sections.
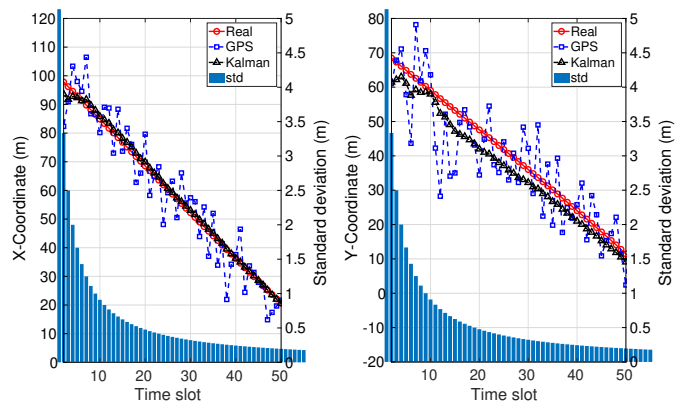

Fig. 1. Kalman-Filter abscissa (left) and ordinate (right) estimations for a standard deviation of $10 \mathrm{~m}$ for the GPS error.

\section{A. Kalman-Filter}

Kalman-Filter is widely used to reduce errors in GPS localization [22]. Kalman-filtering methods consist of twostage recursive equations, namely: prediction and measurement update equations. ECASO adapts the first-stage prediction equations as follows:

$$
\begin{array}{r}
\hat{S}_{k}^{t \mid t-1}=F \cdot \hat{S}_{k}^{t-1}+G \cdot A_{k}^{t} \\
P_{k}^{t \mid t-1}=F \cdot P_{k}^{t-1} \cdot(F)^{T}+Q
\end{array}
$$

where, $S_{k}^{t}$ denotes the system term of interest vector (in our case, it is the UAV's GPS coordinates $\left.\left(x_{k}^{t}, y_{k}^{t}\right)\right), F$ is the state transition matrix, $G$ is the control input matrix, $Q$ is the process noise covariance matrix and $A_{k}^{t}$ the control input system. The matrix $P$ is the state transition error covariance matrix. The second stage Kalman-Filtering measurement equations are given by:

$$
\begin{array}{r}
\hat{S}_{k}^{t \mid t}=\hat{S}_{k}^{t \mid t-1}+K G_{k}^{t} \cdot\left(Z_{k}^{t}-H \cdot \hat{S}_{k}^{t \mid t-1}\right) \\
P_{k}^{t \mid t}=\hat{S}_{k}^{t \mid t-1}-K G_{k}^{t} \cdot H \cdot P_{k}^{t-1 \mid t-1}
\end{array}
$$

where, $Z$ is the GPS measurement vector. The Kalman-Filter gain $K G$ is given by:

$$
K G_{k}^{t \mid t}=P_{k}^{t \mid t-1}+H^{T} \cdot\left[R_{k}^{t}+H \cdot P_{k}^{t-1 \mid t-1}\right]^{-1}
$$

with $R_{k}^{t}$ is the measurement's white noise covariance matrix. The performances of Kalman-Filter are assisted via simulation. The simulation results (See Fig.1) show that Kalman-Filter estimation gets closer to the real UAV localization better than GPS measurement.

\section{B. Optimizer}

The optimization formulated previously is a non-convex, chance-constrained optimization problem, making its resolution challenging and hardly tractable. Thus, the chanceconstrained optimization needs to be transformed into a convex optimization through relaxation. The optimal solution is then derived from the transformed optimization model. Let us consider two UAVs, $U_{k}$ and $U_{l}$. Their respective localization coordinates $L_{k}^{t}=\left(x_{k}^{t}, y_{k}^{t}\right)$ and $L_{l}^{t}=\left(x_{l}^{t}, y_{l}^{t}\right)$ follow a Gaussian distribution; that is, $x_{k}^{t} \sim \mathcal{N}\left(\mu_{k, x}, \sigma_{k, x}^{2}\right), y_{k}^{t} \sim$ $\mathcal{N}\left(\mu_{k, y}, \sigma_{k, y}^{2}\right), x_{l}^{t} \sim \mathcal{N}\left(\mu_{l, x}, \sigma_{l, x}^{2}\right)$ and $y_{l}^{t} \sim \mathcal{N}\left(\mu_{l, y}, \sigma_{l, y}^{2}\right)$. Let $\left(x_{k}^{t+1}, y_{k}^{t+1}\right)$ and $\left(x_{l}^{t+1}, y_{l}^{t+1}\right)$ denote the coordinates of, respectively, $U_{k}$ and $U_{l}$ at time-step $t+1$, where, 
$x_{k}^{t+1}=x_{k}^{t}+v_{k, x}^{t} \cdot \tau ; y_{k}^{t+1}=y_{k}^{t}+v_{k, y}^{t} \cdot \tau ; x_{l}^{t+1}=x_{l}^{t}+v_{l, x}^{t} \cdot \tau ;$ $y_{l}^{t+1}=y_{l}^{t}+v_{l, y}^{t} \cdot \tau$. The coordinates $x_{k}^{t+1}, y_{k}^{t+1}, x_{l}^{t+1}$ and $y_{l}^{t+1}$ follow also a Gaussian distribution with mean $\left(\mu_{k, x}+v_{k, x}^{t} \cdot \tau\right),\left(\mu_{k, y}+v_{k, y}^{t} \cdot \tau\right),\left(\mu_{l, x}+v_{l, x}^{t} \cdot \tau\right)$ and $\left(\mu_{l, y}+v_{l, y}^{t} \cdot \tau\right)$, and standard deviation $\sigma_{k, x}, \sigma_{k, y}, \sigma_{l, x}$ and $\sigma_{l, y}$, respectively.

Let us have the following denotations and assumption:

$$
\begin{aligned}
& x=x_{k}^{t+1}-x_{l}^{t+1} \text { and } y=y_{k}^{t+1}-y_{l}^{t+1} \\
& \mu_{x}=\left(\mu_{k, x}-\mu_{l, x}\right)+\left(v_{k, x}^{t}-v_{l, x}^{t}\right) \cdot \tau \text { with } \mu_{x} \neq 0 \\
& \mu_{y}=\left(\mu_{k, y}-\mu_{l, y}\right)+\left(v_{k, y}^{t}-v_{l, y}^{t}\right) \cdot \tau \text { with } \mu_{y} \neq 0 \\
& \sigma_{x}^{2}=\sigma_{k, x}^{2}+\sigma_{l, x}^{2} \text { with } \sigma_{x} \neq 0 \\
& \sigma_{y}^{2}=\sigma_{k, y}^{2}+\sigma_{l, y}^{2} \text { with } \sigma_{y} \neq 0 \text { and } \sigma_{x}=\sigma_{y} .
\end{aligned}
$$

Based on (3) and (1), the probability of collision will be defined as follows:

$$
P_{r}(\text { collision })=P_{r}\left(x^{2}+y^{2} \leq D_{t h}^{2}\right)
$$

Thus, according to [10], the probability of collision is computed as follows:

$$
P_{r}(\text { collision })=1-\mathcal{Q}_{2}\left(\frac{\lambda}{\sigma}, \frac{D_{t h}}{\sigma}\right)
$$

Subject to:

$$
\begin{gathered}
\sigma^{2}=\sigma_{x}^{2}=\sigma_{y}^{2} \\
\lambda=\sqrt{\mu_{x}^{2}+\mu_{y}^{2}} \\
Q_{2}\left(\frac{\lambda}{\sigma}, \frac{D_{t h}}{\sigma}\right)=\frac{1}{\alpha} \int_{\frac{D_{t h}}{\sigma}}^{\infty} x^{2} \exp \left(-\frac{x^{2}+\left(\frac{\lambda}{\sigma}\right)^{2}}{2}\right) I,\left(\frac{\lambda}{\sigma}\right) x d_{x}
\end{gathered}
$$

Where, $Q_{2}(a, b)$ is the second order Generalized Marcum $Q$ function. Thus, the probability of collision follows a second-degree non-central Chi-square distribution. By substituting (15) in (6), we obtain the following:

$$
1-\mathcal{Q}_{2}\left(\frac{\lambda}{\sigma}, \frac{D_{t h}}{\sigma}\right) \leq \epsilon
$$

Since it is difficult to get closed-form expression for $\lambda$ via derivation for second-order non-central Chi-square distribution. Then, we will first solve numerically (17) to obtain $\lambda$ that allows defining the safety distance threshold that should separate any pair of UAVs. Regardless the literature, ECASO considers that the collision happens probably when the distance between any pair of UAVs $U_{k}$ and $U_{l}$ is less than their dimensions referred by their radius $r_{k}$ and $r_{l}$ where, $D_{t h}=r_{k}+r_{l}$.

$$
1-\mathcal{Q}_{2}\left(\frac{\lambda}{\sigma}, \frac{D_{t h}}{\sigma}\right)=\epsilon
$$

Therefore, a new constraint (18) is added to control the UAVs' motions according to $\lambda$.

$$
\sqrt{\mu_{x}^{2}+\mu_{y}^{2}} \geq \lambda
$$

where $\mu_{x}$ and $\mu_{y}$ include the decision variables of every pair of UAVs. However, constraint (18) is non-convex and it

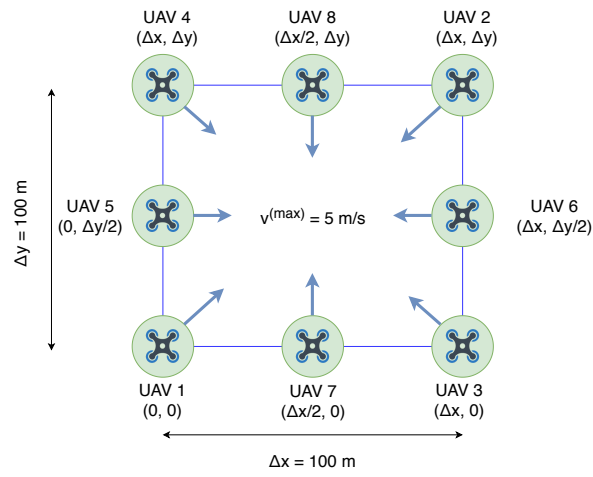

Fig. 2. Experimental setup.

is difficult to get an optimal solution immediately. Thus, the following relaxation is adopted:

$$
\begin{gathered}
\dot{\mu}_{x} \leq \mu_{x}+\mathcal{B}_{\S} \cdot \mathcal{M} \\
\mu_{x} \leq \dot{\mu}_{x}+\mathcal{B}_{\S} \cdot \mathcal{M} \\
\dot{\mu}_{x} \geq 0 \\
\dot{\mu}_{x} \leq-\mu_{x}+\left(1-\mathcal{B}_{\S}\right) \cdot \mathcal{M} \\
-\mu_{x} \leq \dot{\mu}_{x}+\left(1-\mathcal{B}_{\S}\right) \cdot \mathcal{M} \\
\dot{\mu}_{y} \leq \mu_{y}+\mathcal{B}_{\dagger} \cdot \mathcal{M} \\
\mu_{y} \leq \dot{\mu}_{y}+\mathcal{B}_{\dagger} \cdot \mathcal{M} \\
\dot{\mu}_{y} \geq 0 \\
\dot{\mu}_{y} \leq-\mu_{y}+\left(1-\mathcal{B}_{\dagger}\right) \cdot \mathcal{M} \\
-\mu_{y} \leq \dot{\mu}_{y}+\left(1-\mathcal{B}_{\dagger}\right) \cdot \mathcal{M}
\end{gathered}
$$

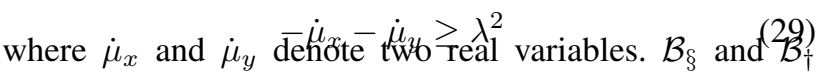
are Boolean variables, and $\mathcal{M}$ tends to infinity. The above constraints ensure the following: constraints (19)-(23) ensure that $-\dot{\mu}_{x} \geq\left|\mu_{x}\right|$; while constraints (24) and (28) ensure that $-\dot{\mu}_{y} \geq\left|\mu_{y}\right|$. Constraint (29) ensures that $\left|\mu_{x}\right|+\left|\mu_{y}\right| \geq \lambda^{2}$.

Indeed, the non-convex chance constraint (6) will be replaced by the equation(17) as well as the constraints (19)(29). Furthermore, since the main objective (4) follows a Gaussian distribution, it could be reformulated as follows:

$$
\begin{array}{r}
\min \sum_{k=1}^{K} \sigma_{k, x}\left(1+\left(x_{k}^{t}-x_{k}^{T}+v_{k, x}^{t} \cdot \tau\right)^{2}\right)+ \\
\sigma_{k, y}\left(1+\left(y_{k}^{t}-y_{k}^{T}+v_{k, y}^{t} \cdot \tau\right)^{2}\right)
\end{array}
$$

Finally, our initial non convex chance-constrained problem (4) will be replaced by the following convex deterministic one:

$$
\begin{array}{r}
\min \sum_{k=1}^{K} \sigma_{k, x}\left(1+\left(x_{k}^{t}-x_{k}^{T}+v_{k, x}^{t} \cdot \tau\right)^{2}\right)+ \\
\sigma_{k, y}\left(1+\left(y_{k}^{t}-y_{k}^{T}+v_{k, y}^{t} \cdot \tau\right)^{2}\right)
\end{array}
$$

Subject to:

(5),(7),(19), (20), (21), (22), (23)

,(24), (25), (26), (27), (28), (29) 


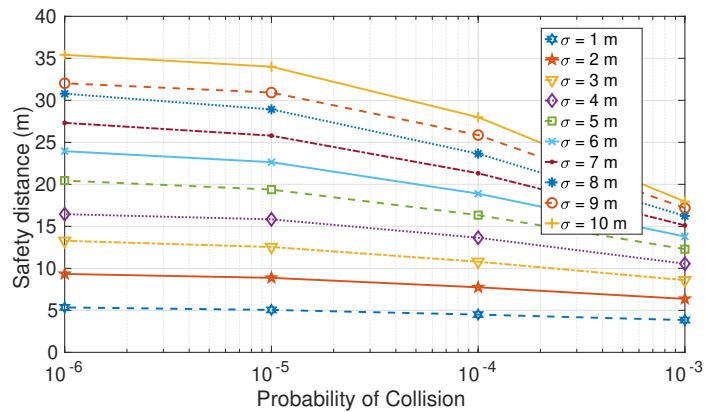

Fig. 3. Safety distance versus the probability of collision.

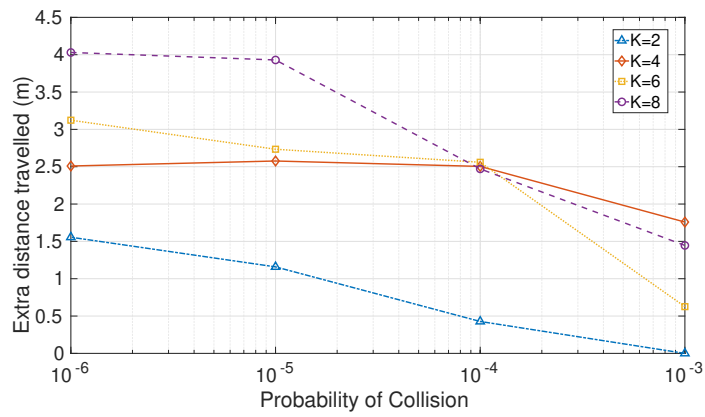

Fig. 4. Extra distance travelled versus the probability of collision $\epsilon$.

\section{Results}

This section includes the description of the experimental setup employed to assess the performance of ECASO along with the discussion of the respective simulation results.

\section{A. Experimental Setup}

In order to evaluate the performance of ECASO we developed a simulator following a fixed-increment time progression. At each time-step the following actions take place: i) the current position provided by the GPS for each UAV $k$ is obtained by sampling a Gaussian distribution, ii) the Kalman-Filter is applied to that data to refine the position estimation, iii) the ECASO optimizer is run to get the optimal velocity components for each UAV (i.e., $v_{k, x}^{t}$ and $v_{k, y}^{t} \forall k \in[1, K]$, and iv) the real position of the UAV is modified according to the velocity vector provided by ECASO for the next time-step. The previous procedure is repeated until the stop condition, which is every UAV got its corresponding target, is reached. It shall be noted that when a given UAV reaches its corresponding target, ECASO excludes it from the optimization process in the subsequent time-steps.

Figure 2 depicts the simulated scenario considered in this work. Specifically, we considered a squared area of $100 \times 100 \mathrm{~m}^{2}$. The initial position of the UAVs is showed in Fig. 2. The vectors in Fig. 2 represent the direction each UAV hat to follow to reach its target position. More precisely, the target positions for UAVs $1,2,3,4,5,6,7$, and 8 are $(\Delta x, \Delta y),(0,0),(0, \Delta y),(\Delta x, 0),(\Delta x, \Delta y / 2)$, $(0, \Delta y / 2),(\Delta x / 2, \Delta y),(\Delta x / 2,0)$, respectively. The max-

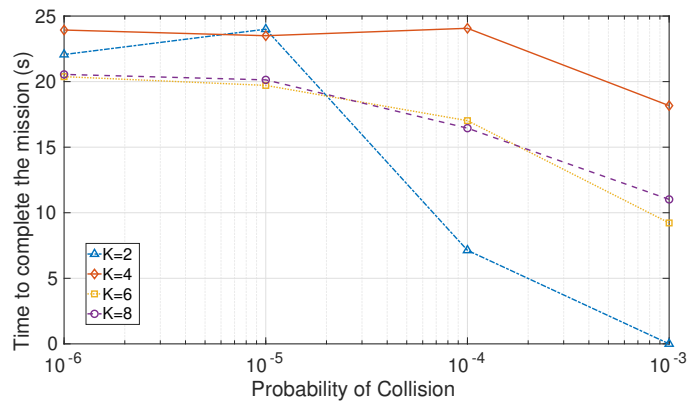

Fig. 5. Time to complete the mission versus the probability of collision $\epsilon$.

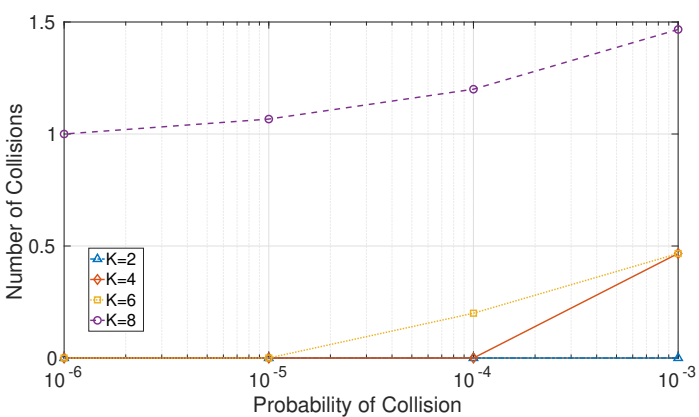

Fig. 6. Number of collisions versus the probability of collision $\epsilon$.

imum velocity considered for every UAV was set to $5 \mathrm{~m} / \mathrm{s}$. Each experiment was run fifteen times.

The different matrices of the Kalman-Filter for each UAV $k$ were initialized as follows:

$$
\begin{aligned}
F & =\left[\begin{array}{cccc}
1 & 0 & \Delta T & 0 \\
0 & 1 & 0 & \Delta T \\
0 & 0 & 1 & 0 \\
0 & 0 & 0 & 1
\end{array}\right] ; \quad G=\left[\begin{array}{cc}
\frac{\Delta T^{2}}{2} & 0 \\
0 & \frac{\Delta T^{2}}{2} \\
\Delta T & 0 \\
0 & \Delta T
\end{array}\right] \\
R & =\left[\begin{array}{cc}
\sigma_{x} & 0 \\
0 & \sigma_{y}
\end{array}\right] ; \quad Q=\mathbf{0}_{4 \times 4} ; \quad K G=\mathbf{0}_{4 \times 2} ; \\
H & =\left[\begin{array}{cccc}
1 & 0 & 0 & 0 \\
0 & 1 & 0 & 0
\end{array}\right] ; \quad P=\left[\begin{array}{cccc}
\frac{\sigma_{x}}{2} & 0 & 0 & 0 \\
0 & \frac{\sigma_{y}}{2} & 0 & 0 \\
0 & 0 & 0 & 0 \\
0 & 0 & 0 & 0
\end{array}\right]
\end{aligned}
$$

where $\mathbf{0}_{m \times n}$ denotes a matrix of all zeros with $m \times n$ elements.

\section{B. ECASO Performance}

Figure 3 depicts the safety distance versus the probability of collision $\epsilon$ for different GPS errors (denoted in the figure as $\sigma$ ). As shown, the safety distance decreases with the probability of collision and increases with the GPS error This suggests a tradeoff between the probability of collision (reliability of the system) and the UAVs autonomy. That is, the lower the probability of collision becomes, the lower will be the UAVs autonomy as it is expected they will travel longer distances. The previous hypothesis is supported by the results shown in Figs. 4, 5, and 6. Interestingly, the results suggest that, for any $\sigma$, the safety distance saturates, i.e., converges to a maximum value, when decreasing the 
probability of collision. Thus, it is possible to configure the system in order to expect near zero collisions, which is an interesting feature for applications that require a high level of reliability. Regarding the GPS error, as expected, the lower it is, the better. This motivates the use of approaches such as Kalman-Filter to improve the accuracy of the estimated position in order to build an energy-efficient system.

Figures 4, 5, and 6 show the impact of the tolerated probability of collision $\epsilon$ and the number of UAVs $K$ involved in a potential situation of collision on the extra distance, time to complete the mission, and number of collisions, respectively. As previously mentioned, these results confirms the lower the $\epsilon$ is, the more reliable will be the system (i.e., lower number of collisions) and the less energy-efficient it will become (i.e., longer traveled distance and time to complete the mission). Besides, the number of UAVs $K$ involved in a potential situation of collision decreases the energy efficiency of the system as it is expected the UAVs will have to do more complex maneuvers to overcome the collisions. In the same, way the probability of collision will increase with the number of UAVs $K$ involved in a potential collision.

\section{CONCLUSION}

In this paper, we have suggested a solution that aims to prevent the collisions among UAVs using a realistic scenario that considers the errors that could happen in the GPS reading. We have considered that the GPS localization error follows a Gaussian distribution that makes our solution considers real scenario when preventing the collisions. We have leveraged the strength of Kalman-Filter technique for reducing the GPS reading errors. The proposed solution uses a chance-constrained optimization technique for preventing the collisions among the UAVs. The obtained results demonstrate the efficiency of proposed solution in terms of execution time and extra distance traveled (i.e., energy). Also, we have shown that the increase in the probability of collision has a positive impact on the former parameters, however it becomes with unavoidable cost, which is the probability of collisions among UAVs.

\section{ACKNOWLEDGMENT}

This work was partially supported by the European Union's Horizon 2020 Research and Innovation Program through the 5G!Drones Project under Grant No. 857031, by the Academy of Finland 6Genesis project under Grant No. 318927, and by the Academy of Finland CSN project under Grant No. 311654.

\section{REFERENCES}

[1] N. H. Motlagh, T. Taleb, and O. Arouk, "Low-altitude unmanned aerial vehicles-based internet of things services: Comprehensive survey and future perspectives," IEEE Internet of Things Journal, vol. 3, no. 6, pp. 899-922, 2016.

[2] Y. K. Kwag and C. H. Chung, "Uav based collision avoidance radar sensor," In Proc. of 2007 IEEE International Geo-science and Remote Sensing Symposium(IGARSS 2007), pp. 639-642, July 2007.

[3] F. Giancarmine, A. Domenico, M. Antonio, C. Ciro, C. Umberto, C. Federico, and L. Salvatore, "Multi-sensor-based fully autonomous non-cooperative collision avoidance system for unmanned air vehicles," Journal of Aerospace Computing Information and Communication, vol. 5, no. 10, pp. 338-360, 2008.
[4] M. C. P. Santos, C. D. Rosales, M. Sarcinelli-Filho, and R. Carelli, "A novel null-space-based uav trajectory tracking controller with collision avoidance," IEEE/ASME Transactions on Mechatronics, vol. 22, no. 6, pp. 2543-2553, Dec 2017.

[5] B. M. Albaker and N. A. Rahim, "A survey of collision avoidance approaches for unmanned aerial vehicles," in 2009 International Conference for Technical Postgraduates (TECHPOS), Dec 2009, pp. $1-7$.

[6] S. Ouahouah and J. Prados-Garzon and T. Taleb and C. Benzaid, "Energy and Delay Aware Physical Collision Avoidance in Unmanned Aerial Vehicles," in Proc. of IEEE Global Communications Conference (GLOBECOM), Dec. 2018.

[7] M. G. Ting-Hua Yi., Hong-Nan Li., "Experimental assessment of highrate GPS receivers for deformation monitoring of bridge," Elsivier, vol. 46, no. 1, pp. 420 - 432, Aug. 2012.

[8] L. Blackmore, M. Ono, and B. C. Williams, "Chance-Constrained Optimal Path Planning with Obstacles," IEEE Transactions on Robotics, vol. 27, no. 6, pp. 1080 - 1094, Dec. 2011.

[9] D. Lyons and J.-P. Calliess and U. D. Hanebeck, "Chance Constrained Model Predictive Control for Multi-Agent Systems with Coupling Constraints," in Proc. of the American Control Conference, Jun. 2012, pp. $1223-1230$.

[10] M. K. Simon, Probability Distributions Involving Gaussian Random Variables: A Handbook for Engineers, Scientists and Mathematicians. Berlin, Heidelberg: Springer-Verlag, 2006.

[11] T. Taleb, K. Ooi, and K. Hashimoto, "An efficient collision avoidance strategy in its systems," In Proc. of IEEE Wireless Communications and Networking Conference, WCNC, Las Vegas, USA, March 2008.

[12] T. Taleb, S. Dutta, A. Ksentini, M. Iqbal, and H. Flinck, "Toward an effective risk-conscious and collaborative vehicular collision avoidance system," IEEE Communications Magazine, vol. 55, no. 3, pp. 38-43, March 17.

[13] R. Sharma and D. Ghose, "Collision avoidance between uav clusters using swarm intelligence techniques," International Journal of Systems Science, vol. 40, no. 5, pp. 521-538, 2009.

[14] J. W. Park, H. D. Oh, and M. J. Tahk, "Uav collision avoidance based on geometric approach," In Proc. of 2008 SICE Annual Conference, pp. 2122-2126, August 2008.

[15] J. P. K. Kim and M. Tahk, "Uav collision avoidance using probabilistic method in 3-d," In Proc. of 2007 International Conference on Control, Automation and Systems, pp. 826-829, August 2007.

[16] M. Shanmugavel, A. Tsourdos, and B. A. White, "Collision avoidance and path planning of multiple uavs using flyable paths in 3d," in 2010 15th International Conference on Methods and Models in Automation and Robotics, Aug 2010, pp. 218-222.

[17] Z. Chao, L. Ming, Z. Shaolei, and Z. Wenguang, "Collision-free uav formation flight control based on nonlinear mpc," in $2011 \mathrm{In}$ ternational Conference on Electronics, Communications and Control (ICECC), Sept 2011, pp. 1951-1956.

[18] J. G. Manathara and D. Ghose, "Reactive collision avoidance of multiple realistic uavs," Aircraft Engineering and Aerospace Technology, vol. 83, no. 6, pp. 388-396, 2011. [Online]. Available: https://doi.org/10.1108/00022661111173261

[19] L. A. Tony, D. Ghose, and A. Chakravarthy, "Avoidance maps: A new concept in uav collision avoidance," in 2017 International Conference on Unmanned Aircraft Systems (ICUAS), June 2017, pp. 1483-1492.

[20] R. He, R. Wei, and Q. Zhang, "Uav autonomous collision avoidance approach," Automatika, vol. 58, no. 2, pp. 195-204, 2017. [Online]. Available: https://www.tandfonline.com/doi/abs/10.1080/00051144.2017.1388646

[21] Y. Lin and S. Saripalli, "Sampling-based path planning for uav collision avoidance," IEEE Transactions on Intelligent Transportation Systems, vol. 18, no. 11, pp. 3179-3192, Nov 2017.

[22] R. Faragher, "Understanding the basis of the kalman filter via a simple and intuitive derivation [lecture notes]," IEEE Signal Processing Magazine, vol. 29, no. 5, pp. 128-132, Sep. 2012. 